\title{
The Facial Adipose Tissue: A Revision
}

\author{
Ilja Kruglikov, PhD ${ }^{1}$ Oscar Trujillo, MD $^{2}$ Quick Kristen ${ }^{3}$ Kerelos Isac $^{3} \quad$ Julia Zorko ${ }^{3}$ Maria Fam ${ }^{3}$ \\ Kasie Okonkwo ${ }^{3}$ Asima Mian ${ }^{3}$ Hyunh Thanh ${ }^{3}$ Konstantin Koban, MD ${ }^{4}$ \\ Anthony P. Sclafani, MD, FACS ${ }^{2}$ Hanno Steinke, PhD $^{5}$ Sebastian Cotofana, MD, PhD 3,6
}

${ }^{1}$ Scientific Department, Wellcomet GmbH, Karlsruhe, Germany

2 Department of Otolaryngology-Head and Neck Surgery,

Weill Cornell Medical College, New York, New York

3 Department of Anatomy, Ross University School of Medicine,

Roseau, West Indies

${ }^{4}$ Department of Hand, Plastic and Aesthetic Surgery, Ludwig-

Maximilians University, Munich, Germany

${ }^{5}$ Department of Medicine, Institute of Anatomy, University of Leipzig,

Leipzig, Germany

${ }^{6}$ Institute of Anatomy, Paracelsus Medical University Salzburg and

Nuremberg, Salzburg, Austria

Facial Plast Surg 2016;32:671-682.

\author{
Address for correspondence Sebastian Cotofana, MD, PhD, \\ Department of Anatomy, Ross University School of Medicine, P.O. Box \\ 266, Roseau, Commonwealth of Dominica, West Indies \\ (e-mail: sebastiancotofana@rossu.edu).
}

\begin{abstract}
Keywords

- facial fat

- fat compartments

- dermal adipose tissue

- subcutaneous adipose tissue

Recent advantages in the anatomical understanding of the face have turned the focus toward the subcutaneous and deep facial fat compartments. During facial aging, these fat-filled compartments undergo substantial changes along with other structures in the face. Soft tissue filler and fat grafting are valid methods to fight the signs of facial aging, but little is known about their precise effect on the facial fat. This narrative review summarizes the current knowledge about the facial fat compartments in terms of anatomical location, histologic appearance, immune-histochemical characteristics, cellular interactions, and therapeutic options. Three different types of facial adipose tissue can be identified, which are located either superficially (dermal white adipose tissue) or deep (subcutaneous white adipose tissue): fibrous (perioral locations), structural (major parts of the midface), and deposit (buccal fat pad and deep temporal fat pad). These various fat types differ in the size of the adipocytes and the collagenous composition of their extracellular matrix and thus in their mechanical properties. Minimal invasive (e.g., soft tissue fillers or fat grafting) and surgical interventions aiming to restore the youthful face have to account for the different fat properties in various facial areas. However, little is known about the macro- and microscopic characteristics of the facial fat tissue in different compartments and future studies are needed to reveal new insights to better understand the process of aging and how to fight its signs best.
\end{abstract}

Comparing the histologic characteristics of the hypodermis amongst mammal species, different layered arrangements can be identified. Apes and dedicated furred mammal species display in their hypodermal fat layer a fibrous and/or muscular layer located between the dermis and the deep fascia ( - Fig. 1). This specific layer is called the panniculus carnosus and separates the subcutaneous fat into a superficial and a deep fat layer (-Fig. 1A). In other mammals such as pigs (sus scrofa domestica) that lack fur, this layer is absent and the total hypodermal fat layer is called panniculus adiposus (-Fig. 1B). In the latter arrangement, the perforating arteries can reach the skin and can regulate the dermal blood flow and thus skin temperature and nutrition. This different layered arrangement of the subcutaneous fat is believed to provide an evolutionary advantage to fur-less mammals (e.g., the human) as they are capable of adapting to different climate conditions based on
Issue Theme Worldwide Perspectives in Facial Plastic Surgery; Guest Editor, Fred G. Fedok, MD, FACS
Copyright (c) 2016 by Thieme Medical Publishers, Inc., 333 Seventh Avenue, New York, NY 10001, USA. Tel: +1(212) 584-4662.
DOI http://dx.doi.org/ 10.1055/s-0036-1596046. ISSN 0736-6825. 




Fig. 1 Different types of skin. (A) Arrangement of the human skin in the head and neck region consisting of (1) dermis, (2) superficial fat (here dermal white adipose tissue [dWAT]), (3) panniculus carnosus (here superficial musculoaponeurotic system [SMAS]), (4) deep fat (here subcutaneous white adipose tissue [swat]), (5) deep fascia/periosteum, and (6) deep structures (here muscle). (B) Normal arrangement of skin in most mammals consisting of (1) dermis, (2) panniculus adiposus (here sWAT), (3) deep fascia/periosteum, and (4) deep structures here muscle.

their advanced thermoregulatory capacity whereas other mammals cannot. ${ }^{1}$

It is widely believed that the human hypodermal fat layer is absent of the panniculus carnosus. Investigating the threedimensional (3D) functional anatomy of the human adipose tissue $(\mathrm{AT})^{2,3}$ revealed a fascial plane composed of fibrous connective tissue and muscular fibers, which was identified in the hypodermal plane and can be regarded as the homologue to the panniculus carnosus in rodents. ${ }^{2}$ In the head this separating layer is called galea aponeurotica; in the face, superficial musculo-aponeurotic system (SMAS); in the neck, platysma; in the abdominal region, Scarpas' fascia; in the perineum, Colles' fascia; and in the scrotum, Dartos tunic.

It has been shown that this hypodermal fascial arrangement subdivides the subcutaneous fat in the abdominal ${ }^{3}$ and in the facial $^{4}$ regions into superficial and deep fat with different morphologic characteristics. To better understand this arrangement and the distribution of the white adipose tissue (WAT) in the face, we critically discuss here the recent insights in this field.

\section{Anatomy of Various Facial Fat Compartments}

\section{General Arrangement}

The face has a layered structure that can be described from superficial to deep as follows: layer 1: skin; layer 2: subcutaneous fat; layer 3: superficial musculoaponeurotic system (SMAS); layer 4: deep fat; and layer 5: deep fascia or periosteum. These five layers can be found throughout the face and are continuous from the head into the neck. ${ }^{5}$ From here two different compartmentalized layers of fat are continuously present in the face: superficial and deep. Of those, the superficial layer can be subdivided further into two different arrangements with different microstructure: in perioral, nasal, and in parts of periorbital areas versus the midface area. The deep layer itself can be differentially classified into the deep fat compartments and the buccal fat pad. These different facial fat arrangements have different functions and are characterized by different types of AT including its proper extracellular matrix (ECM).

\section{Superficial Fat Compartments (Layer 2)}

The subcutaneous fat layer contains dermal white adipose tissue (dWAT) and varies in thickness between individuals of different age and ethnicity. It is subdivided by fibrous septae into distinct compartments, which have been previously identified both in cadaveric ${ }^{6-8}$ and imaging studies. ${ }^{9}$ Within the walls of the bounding septae, nerves and vessels traversing this plane can be identified. ${ }^{10}$ Based on cadaveric dissections ${ }^{5}$ and on previous histologic analyses, ${ }^{11}$ two different types can be identified according to Ghassemi et al. ${ }^{11}$ Type 1 can be found in the medial and lateral midface and in parts of the periorbital region, as well as in the temple, forehead, and neck. Here, the adherence of the underlying structures to the skin is loose, and an easy separation of the skin from the underlying fat layer can be performed during anatomical dissections (-Fig. 2A). The WAT in these areas can be classified as structural WAT with a meshwork of fibrous septa enveloping lobules of fat cells that act as small pads with specific viscoelastic properties. ${ }^{12}$ Type 2 can be found in perioral and nasal areas, as well as in the area of the eyebrows. Here, a strong linkage is present between the facial muscles, the collagenous meshwork surrounding the adipocytes and the skin. A separation using blunt dissections is 



Fig. 2 Different types of subcutaneous fatty arrangement as observed during anatomical dissections. (A) Black arrows indicate the location of the labiomandibular sulcus. (B) After removal of skin and reflections of the subcutaneous tissue, the two different types of subcutaneous arrangement can be observed: Red circle: structural white adipose tissue corresponding to Ghassemi-type 1; blue circle: fibrous white adipose tissue corresponding to Ghassemi-type 2.

difficult in these areas, whereas collagenous and muscular fibers directly insert into the skin and connect the skin to the underlying muscles of facial expression (-Fig. 2B). The WAT here can be classified as fibrous with a meshwork of intermingled collagen and elastic fibers as well as muscle fibers. The latter reach up to the dermis of the skin and fat cells are interposed between this collagen fiber-muscle meshwork. ${ }^{12}$ The most prominent demarcation lines between these two subcutaneous arrangements include the nasolabial sulcus, the labiomandibular sulcus, and the submental sulcus (-Fig. 3). Within the midface, the type 1 subcutaneous arrangement results in the formation of the nasolabial, medial, middle, lateral, and buccal subcutaneous fat compartments that have been previously introduced. ${ }^{6,7}$ It is worth mentioning that the medial subcutaneous fat compartment in layer 2 of the midface is also called the malar fat pad by some authors. ${ }^{5,13}$ On the forehead, three superficial fat compartments have been described previously ${ }^{8,9}$ and their description is in line with the arrangement of the fat layers in the midface.

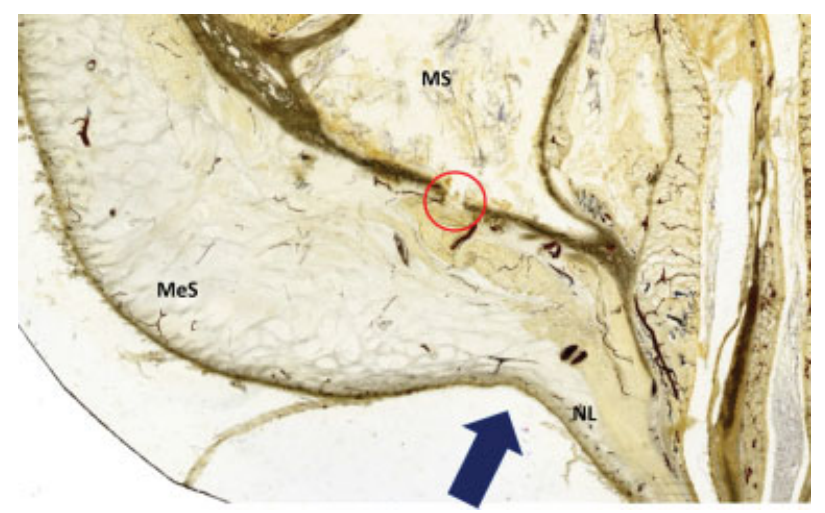

Fig. 3 Histologic cross section of the midface in a transverse plane at the level of the infraorbital foramen (red circle) and the maxillary sinus (MS). The uppermost beginning of the nasolabial sulcus is indicated by the blue arrow. The medial subcutaneous (MeS) and the superficial nasolabial (NL) fat pads are indicated.

\section{Deep Fat Compartments (Layer 4)}

The deep fat layer contains subcutaneous white adipose tissue (sWAT) and is separated from the more superficially located dWAT (layer 2) by the SMAS in the midface, platysma in the neck, and superficial temporal fascia in the temple. The orbicularis oculi and the frontalis muscle can also be identified within this layer as they are continuous with each other on the lower and the lateral forehead. The deep fat layer is likewise separated by fibrous septae into distinct compartments, and similar to the dWAT, these septae serve as transition pathways for the branches of the facial nerve ${ }^{14-16}$ or for branches of the facial artery and vein. ${ }^{16}$ Boundaries of deep fat compartments can also be formed by the origins of muscles of facial expression and their respective bony origins. ${ }^{5}$ The deep fat compartments have been recently described both in cadaveric ${ }^{17-20}$ and in imaging studies. ${ }^{21}$ The deep medial cheek fat compartment is enclosed by the levator labii superioris aleque nasi and the levator anguli oris muscle and lies in close proximity to the infraorbital foramen. ${ }^{21}$ The triangular-shaped deep lateral cheek fat compartment is bounded medially by the facial vein, laterally by the zygomaticus major muscle, and superiorly by the zygomatic ligament. ${ }^{5}$ Medial to the deep medial cheek fat and lateral to the pyriform aperture the deep pyriform space can be identified, which previously was named Ristow-space. ${ }^{22}$ Another fat compartment (so-called deep nasolabial compartment) described both clinically and anatomically lies superficially to the levator labii superioris aleque muscle within the premaxillary space but deep to the orbicularis oculi muscle. Superior to the zygomatic ligament but inferior to the orbicularis retaining ligament and lateral to the facial vein, the suborbicularis oculi fat (SOOF) compartment can be identified, which lies deep to the orbicularis oculi muscle..$^{20,21,23}$ In the supraorbital region on the lateral margin of the orbit, the retroorbicularis fat compartment (ROOF) is located, which is bounded medially by the neurovascular bundle emerging from the supraorbital foramen. In the lateral midface superficial to the masseter muscle, three distinct compartments can be identified, which provide avascular dissection pathways 
during face-lifting procedures: inferior, medial, and superior premasseter compartments. $^{15}$

\section{Deepest Fat Compartments (Deep to Layer 5)}

Comparing with layers 2 and 4 , the deepest fat layer is not continuous, but its fat compartments are few in numbers and the physiologic role of the fat within these compartments has not yet been fully elucidated. The buccal fat pad was first described in $1801^{24}$ and can be classified as such and is regarded as WAT of the deposit or metabolic type. ${ }^{12}$ It is located between the fascia of the buccinator muscle and the fascia of the masseter muscle in the buccotemporal space. ${ }^{25}$ The buccal fat is unique in humans and absent in most of ape species and has four extensions: buccal, pterygoid, pterygopalatine, and temporal; the latter one is also known as the deep temporal fat pad of the temporal region and is found between the deep lamina of the deep temporal fascia and the temporalis muscle. ${ }^{26}$ However, another fat compartment in the deepest layer, which is much less well understood, is the superficial temporal fat pad (layer 6), and is found between the superficial and the deep lamina of the deep temporal fascia, superiorly to the zygomatic arch. ${ }^{26}$ While the superficial lamina of the deep temporal fascia is continuous anteriorly with the orbital septum ${ }^{27}$ but not with the periosteum in the infraorbital region, it provides a space for fat within the prezygomatic space. ${ }^{9,23,27}$ This compartment lies deep to the SOOF and is separated from it by the superficial lamina of the deep temporal fascia.

\section{Facial White Adipose Tissue}

\section{Subcutaneous White Adipose Tissue}

As outlined previously, the facial sWAT can be further refined taking into account that the anatomically identified superficial and deep fat compartments are significantly different both morphologically and physiologically in respect to their WATs.

Anatomically, the sWAT of the head and neck can be classified into two separate WAT depots. As it was mentioned previously, the interface between these WAT depots (superficial versus deep) in humans is less pronounced compared with rodents. Dynamically, deep sWAT is considered a slowly renewal tissue with characteristic turnover times of around 10 years. ${ }^{28,29}$

\section{Dermal White Adipose Tissue}

Recently, it was demonstrated that adipocytes can behave dynamically in the superficial area of sWAT underlying the reticular dermis, where these cells can be involved in such processes as hair follicle cycling, ${ }^{30}$ wound healing, ${ }^{31}$ thermoregulation, ${ }^{32,33}$ and innate immune response of the skin. ${ }^{34}$ In all these processes, dermal adipocytes demonstrate the characteristic remodeling times of days to weeks. It was also assumed that these cells can be involved in spatially local cross-talk with skin cells leading to different inflammatory $^{35-37}$ and pigmentary ${ }^{35}$ skin conditions, as well as in skin aging. ${ }^{38}$ There are actually no genetic tools to selectively manipulate these subdepots since currently there is a lack of specific markers to differentiate between the superficial and deep adipocytes. At the same time, the ability of superficial adipocytes to undergo different phenotypic transformations $^{35}$ (among them the recently discovered adipocytemyofibroblast transition, ${ }^{39}$ which is now believed to play an important role in cutaneous fibrosis) clearly demonstrates the phenotypic plasticity of adipocytes from the dWAT layer. As a logical consequence, it was proposed to introduce dWAT as a special AT depot ${ }^{40,41}$ to differentiate between dermal adipocytes and adipocytes from a deep sWAT.

Furthermore, adipocytes from dWAT can locally react to internal and external factors with a quick modification in their number and cell volumes demonstrating either expansion or reduction of the dWAT layer ( - Fig. 4). Resulting total modulation of WAT volume can many times exceed any effect that can be reached through a structural modification of the much thinner dermis. This evidently shifts the point of interest in noninvasive and minimal invasive aesthetic procedures from fibroblasts to adipocytes. ${ }^{42}$ At the same time, dWAT can be structurally and metabolically heterogeneous on the mesoscopic scale, which is an intermediate between the macroscopic scale of a single fat compartment and microscopic scale presented by single adipocytes or adipocyte clusters. Consequently, modification of this tissue by different internal or external factors can produce a spatial mosaic skin structure. ${ }^{36,37,43}$

In humans, the dWAT does not produce the simple layers of adipocytes running almost parallel to the skin surface as in rodents but additionally demonstrate the protrusions into the dermis usually concentrated around the pilosebaceous units. ${ }^{35}$ A similar structure of dWAT was shown to be typical also for the porcine skin. ${ }^{44}$ Such dWAT structure provides spatially local clusters of dermal adipocytes, which, in an extreme case, can reach the papillary dermis and thus build the anatomical structures that can directly absorb the UV irradiation making dWAT equivalent to a potential target in the pathophysiology of the skin aging. ${ }^{38}$

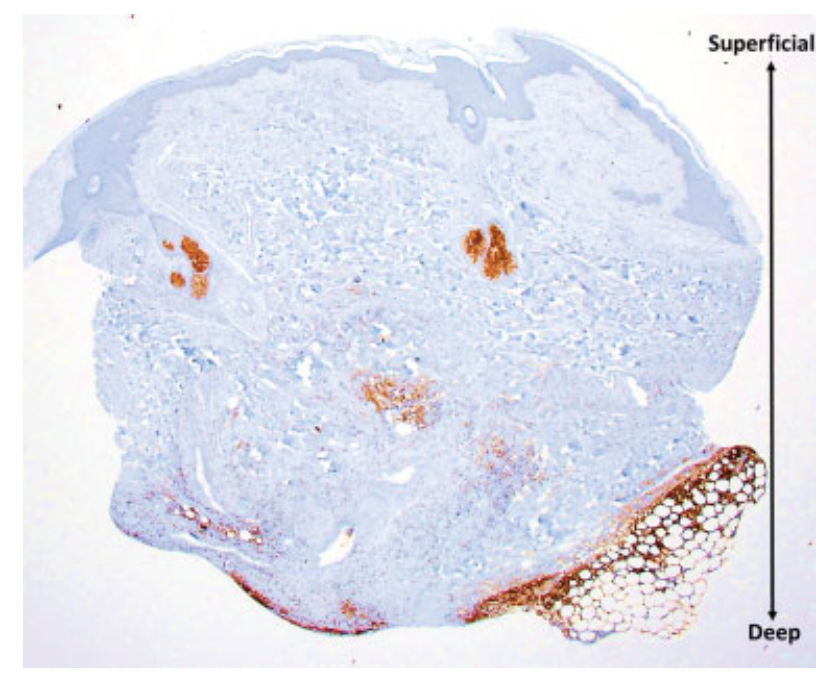

Fig. 4 Immunohistochemical staining with adipophilin of skin biopsy 19 days after the treatment with platelet-rich fibrin matrix showing differentiating dermal white adipocytes (red circles) within dermal white adipose tissue (dWAT). Note: Adipophilin is a protein associated with differentiating adipocytes. 


\section{Microscopic Heterogeneity of Adipose Tissue}

Thus far, macroscopic anatomical description does not permit the differentiation of AT depots according to their structural components such as adipocytes and ECM. At the same time, recent experimental data have demonstrated that the AT structure is extremely heterogeneous on the microscopic and mesoscopic scales and that morphologic peculiarities and physiologic states of different AT depots are strongly dependent on the relative weighting of these components in a given AT volume. Adipocytes were for a long time considered to be the predominant structural component of every AT depot being responsible both for its physiologic properties and mechanical behavior as well as for its reaction to different external factors. This picture was strongly modified during the recent past as it became clear that other structural components of AT-especially, its ECM-are more important for the mechanical stability and renewal dynamics of ATs.

\section{Adipocytes}

Despite the fact that mature adipocytes occupy the main volume of AT, the number of these cells was estimated to be less than $20 \%$ of the total cellularity in ATs. ${ }^{45}$ Partial volume of every AT compartment depends on its cellularity and the characteristic adipocytes' sizes, which vary significantly among different AT depots and have been shown to demonstrate sexual dimorphism. ${ }^{46-48}$ Adipocytes' sizes in any AT depot have a typical bimodal distribution indicating the existence of at least two different subpopulations of small and large adipocytes. ${ }^{49}$ These distributions generally have high coefficients of variation of up to $50 \%$. For example, it was shown that the patients with a body mass index (BMI) less than $25 \mathrm{~kg} / \mathrm{m}^{2}$ have $0.57 \pm 0.23 \mu \mathrm{g}$ of triglycerides per cell in femoral, $0.48 \pm 0.15 \mu \mathrm{g}$ in gluteal, and $0.41 \pm 0.20 \mu \mathrm{g}$ in abdominal areas, respectively. In patients with BMI greater than $30 \mathrm{~kg} / \mathrm{m}^{2}$, these values were shown to be $0.83 \pm 0.18$, $0.71 \pm 0.23$, und $0.78 \pm 0.24 \mu \mathrm{g}$, respectively. ${ }^{46}$

Generally, the volume of any AT depot can be modulated in two different ways: through hyperplasia or hypertrophy, wherein hyperplasia is currently believed to be more characteristic in sWAT and hypertrophy is more often associated with the visceral fat depots. ${ }^{50}$ The hypertrophy of adipocytes was shown to be the first reaction to a high-fat diet, ${ }^{51}$ whereas the hyperplasia normally appears later and is connected with a prolonged excessive feeding. Physiologically small and large adipocytes are different, ${ }^{52}$ and it is believed that small adipocytes are mainly connected with development of new adipocytes from preadipocytes, which is required for good vascularization of AT depots. Adipocytes' sizes can significantly differ in various body and facial areas, ${ }^{4}$ which can reflect different metabolic activities and fat structures in different facial AT compartments.

\section{Extracellular Matrix in Adipose Tissues: Collagen}

Adipocytes are imbedded in the extracellular matrix (ECM) of a special structure containing different types of collagen. The main function of this matrix was long believed to be the mechanical support: during AT expansion or involution, this matrix should be able to modify its structure to accommodate the altered adipocytes. This simple picture was substantially modified during the last years as it became clear that ECM in AT is not a passive scaffold but an active player in different morphologic and physiologic processes, ${ }^{53-56}$ and that its specific modification can even be a hallmark of metabolically changed adipocytes.

The role of collagen in ECM structure of ATs was long underestimated, until it was ascertained that different types of WAT have not only quantitative, but also dramatic qualitative differences in their collagen content. For example, AT of a hypertrophic type containing predominantly large adipocytes demonstrates also high content of collagens (Col) IV and VI, which are the nonfibrillar collagens providing the pericellular fibrosis around the mature adipocytes. ${ }^{57,58}$ Content of $\mathrm{Col} \mathrm{VI}$ in ATs demonstrates sexual dimorphism and varies significantly between different ethnic groups being higher in Asian Indians than in Caucasians. ${ }^{57}$ This fibrosis is supposed to restrict the unlimited expansion of adipocytes, which can lead to their damage and death, and this collagen was shown to be involved in different AT pathologies. Col VI content increases significantly with rising BMI; while the total fibrotic volume in sWAT is almost independent of BMI, ${ }^{58}$ the pericellular fibrosis increases with BMI by three to seven times. ${ }^{59,60}$ Contrarily to the pericellular fibrosis, the intercellular fibrosis exists in the form of thick collagen bundles consisting mainly of $\mathrm{Col}$ I and III that can penetrate the AT in different directions and can span many cell lengths. ${ }^{58}$ Both relative and absolute content of intercellular and pericellular fibrosis in different facial AT compartments can vary significantly.

\section{Extracellular Matrix in Adipose Tissues: Hyaluronic Acid and Water Retention}

Water content in an AT is normally less than $20 \%$, whereas 14 to $16 \%$ of this water is located extracellular and only 2 to $6 \%$ intracellular. These values can be changed dramatically in a hypertrophic fat tissue, which normally demonstrates a significantly increased (up to $50 \%$ in extracellular space and up to four times in the pericellular areas) concentration of hyaluronic acid (HA). ${ }^{61}$

It was proposed that such high water retention around adipocytes prohibits the lipolysis, as the transport of water and glycerin in these cells should be realized through the same aquaporin 7 (AQP7) membrane channels. ${ }^{62}$ Though adipocytes have almost no cytoskeleton and thus appear nearly spherically in vitro, they can respond to a high osmotic pressure only with a limited expansion associated with a risk of the rupture of their cell membranes. This effect should force adipocytes to reduce the number of active AQP7 channels in the aqueous environment, which should consequently reduce the lipolytic activity of adipocytes. Such reduction in AQP7 channels in hypertrophic adipocytes was indeed observed in patients with high BMI values. ${ }^{63}$ Moreover, a depletion of HA content in AT through application of hyaluronidase was able to decrease the fat mass up to $35 \%$, and this reduction was primarily connected with a reduction in the adipocytes' sizes and not with a cell death, thus demonstrating a stimulation of the physiologic activity of adipocytes ${ }^{64-66}$ HA found in a hypertrophic AT has 
a molecular weight of less than $15 \mathrm{kDa} .{ }^{61}$ It is known that the HA fragments of low molecular weight can stimulate the production of tissue fibrosis ${ }^{67}$ and that HA distribution in AT spatially correlates with appearance of fibrotic structures in this tissue. ${ }^{68}$ Moreover, HA was shown to interact with Col VI promoting its assembling in vitro. ${ }^{69}$ From this point of view, HA content can play a pivotal role in a local fibrosis and differential modification of mechanical properties of AT in different facial AT compartments.

\section{Microstructural Classification of Facial Adipose Tissue Depots}

Whereas the macroscopic compartmental classification of facial AT can be seen as state of the art in facial aging, ${ }^{70}$ a microstructural modification of different fat compartments in an aging face has not been described properly, although some authors indeed tried to make the differentiation.

The first attempt to describe the microscopic differences in the structure of various facial fat compartments taking into account both the morphology of adipocytes and the local collagen content was undertaken only recently. The background for this description was proposed in Sbarbati et al, ${ }^{71}$ who subdivided the sWAT depots using the results of transmission electron microscopy and scanning electron microscopy into three groups:

1. Deposit (metabolic) WAT: A nonlobular AT containing large adipocytes and characterized by extremely weak or even absent collagen network that does not wrap the single adipocytes.

2. Structural WAT: An AT containing large adipocytes, whereas each cell is evidently covered by a thin fibrous shell.

3. Fibrous WAT: An AT containing smaller adipocytes, whereas each cell is covered by a thick fibrous shell. This WAT was further subdivided into the lobular and nonlobular fibrous sub-types.

Based on these results, Bartossi et al proposed a classification of the fat compartments from the third medium of the face. $^{12}$ These authors investigated the malar, periorbital, labial, nasal, and buccal fat pads using the same methods as Sbarbati and colleagues ${ }^{71}$ and have shown that

- Malar fat contains the lobules of large mature adipocytes homogeneously covered by thin collagen fibers as well as some newly formed adipocytes located near the capillaries (corresponding to the type 1 characterization of Ghassemi et $\mathrm{al}^{11}$ ).

- Periorbital fat demonstrates the pronounced lobular structure with a dense network of thin fibers around the lobules producing the basket-like structures.

- Labial and nasal fat contains predominantly mature adipocytes embedded in a dense collagen matrix (corresponding to the type 2 characterization of Ghassemi et $\mathrm{al}^{11}$ ).

- Buccal fat is characterized by large mature adipocytes that are not completely covered by a collagen network.
Following the classification proposed by Sbarbati et al, ${ }^{71}$ malar and periorbital fat pads were classified as WAT of the structural type, labial and nasal fat pads as WAT of the fibrous type, and buccal fat pad as WAT of the deposit type. ${ }^{12}$ This classification demonstrates that even the neighboring facial AT compartments can be structurally different, which can not only significantly influence the mechanical properties of the fat pads in single compartments but also mechanically stress the boundaries between these compartments produced by septa, ${ }^{8,18}$ and thus cause a structural modification of the adjacent dermis area. However, it has to be noted that to date not all facial fat compartments have been investigated and detailed information is still missing. Future research will be needed to elaborate the precise characteristics of the remaining uninvestigated fat compartments like the superficial temporal fat pad or the fat within the prezygomatic space. ${ }^{13,27}$

\section{Role of Adipocytes and Fibrotic Structures in Mechanical Properties of Adipose Tissue Depots}

Mechanical properties of AT depots play a pivotal role in the anatomy of the aging face. Taking into account that different facial AT compartments have different morphologic structures (especially concerning the content of pericellular and intercellular fibrosis and the average adipocytes' sizes), it is important to understand how these parameters can modify the stiffness of specific AT depots.

Micromechanically, an AT depot can be described as a closed-cell foam structure. $^{72,73}$ Because elastic shear modulus of triglycerides is much less than that of an $\mathrm{AT}^{72}$ contribution of lipids into mechanical stiffness of sWAT can be neglected, even though they occupy the main tissue volume. Mechanical characteristics of such system are mainly dependent on the thickness of pericellular fibrotic structure containing Col IV and $\mathrm{VI},{ }^{72}$ which can vary significantly depending on the type of $\mathrm{AT}^{74}$ Contrarily to the pericellular fibrosis, the intercellular fibrosis has a coarse-mesh structure with a typical unit size of several millimeters and its contribution to the total mechanical stiffness of AT depot is low. Mechanical strength of an AT depot without Col VI was recently measured to be approximately $50 \%$ lower than that of corresponding AT depot containing Col VI. ${ }^{75}$

On the other hand, as it was shown by Kruglikov ${ }^{73}$ that local mechanical stiffness of AT depot is inversely dependent on the average cell size, being lower for the population of large adipocytes. Remarkably, this parameter is almost independent of the dispersion of adipocytes' sizes. In other words, only the average cell size and not the cell size distribution is important for the mechanical stiffness of AT. Additionally, local appearance of small adipocytes (e.g., through induced adipogenesis or lipolysis) should improve the mechanical characteristics of the given AT depot. Moreover, an AT containing two different subpopulations of adipocytes of very different sizes can demonstrate a spatial heterogeneity of its mechanical properties.

According to the microstructural classification of the facial AT compartments provided in Bartossi et al, ${ }^{12}$ the "fibrous WAT" should have the highest content of pericellular fibrotic structures and thus the highest mechanical stiffness. On the 
other hand, the "deposit WAT" is characterized by almost completely absent pericellular fibrotic structures and thus should have the worst mechanical properties.

Though every facial AT compartment undergoes a specific modification of its cellular and fibrotic components in aging, these changes can be very different even in neighboring facial compartments that can significantly influence the total facial aging appearance. For example, increase in the difference in mechanical modules in the neighboring compartments can produce a mechanical pressure on the septa between these facial areas leading to its deformation and consequent weakening in an aging face. Such deformation should be especially strong for the boundaries between AT compartments with sufficiently different pericellular structures. This effect can be clinically observed in the nasolabial and labiomandibular sulcus. Medial to both of the sulci, fibrous WAT can be identified, whereas lateral to them, structural WAT is present. During aging, the mechanical properties change and lead (along with other factors) to a bulging of the less mechanically stable tissue following gravity.

\section{Facial Aging on Mesoscopic Scale}

Present theories of skin aging almost completely exclude the involvement of AT in this process. At the same time, significant modification of dWAT was observed both in intrinsically ${ }^{51,76}$ and extrinsically ${ }^{77,78}$ aged murine skin. Reduction in the dWAT in a chronically UV-irradiated skin was shown to correlate with replacement of this volume by fibrotic structures indicating the involvement of recently revealed effect of adipocyte-myofibroblast transition, ${ }^{39}$ which mainly involves the dermal adipocytes. ${ }^{35,38}$ The appearance of skin wrinkles can be connected with a local disappearance of dermal adipocytes and deposition of fibrotic structures under the location of a skin depression.

Although sWAT and dermis appear anatomically as absolutely different tissues with a clearly defined boundary, there are several indications that these tissues strongly interact with each other. Co-culturing of adipocytes with fibroblasts demonstrated that hypertrophic (enlarged) adipocytes could significantly suppress the synthetic activity of fibroblasts through release of free fatty acids, whereas the small adipocytes had no such effect. ${ }^{79}$ Recently it was shown that the increment in sWAT correlates with decrease in elastic fibers' content in the dermis and that this degradation can be connected with significantly elevated activity of matrix metalloproteinases. ${ }^{80}$ Consequently, not only the effects of chronological and photo-induced aging but also the expansion of sWAT can induce a relatively quick degradation of elastic fibers in the dermis, which, under normal conditions, should persist in this tissue for many decades. ${ }^{81-83}$ It can be speculated whether a local modulation of a superficial sWAT layer can lead to a corresponding response in an adjacent dermal area, leading to a change of its mechanical characteristics on the mesoscopic scale. Macroscopically, such negative adipocytefibroblast interaction can significantly influence the sagging severity in lower cheek ${ }^{84,85}$ and the formation of the nasolabial fold, ${ }^{86}$ both contributing to the formation of the aging face.
Another anatomical basis for wrinkles, which involves a specific fat depot, was recently proposed by Pessa et al. ${ }^{87}$ These authors found a spatial correlation between dedicated wrinkles (forehead crease, crow's foot, and nasojugal crease) and the location of the big lymphatic vessels surrounded by the perilymphatic adipose tissue (PLAT). It is known that defective lymphatic transport can result in significant expansion of the $\mathrm{AT}^{88}$; at the same time, it is not actually known whether modulation of PLAT can influence the local lymph transport. This raises the question whether the small, superficial PLAT depot located between well-known large facial AT compartments should be considered as a separate fat entity with the capability of influencing the process of facial aging.

\section{Soft Tissue Fillers Targeting Facial Adipose Tissue}

Application of soft tissue fillers (SFTs) is a broadly accepted minimally invasive antiaging procedure. Long-termed skin improvement after injection of different types of SFTs was theoretically connected with activation of the neocollagenesis in dermis, which was criticized as an unrealistic mechanism. ${ }^{83,89}$ Independent of the filler type, STFs are generally applied into the deeper located sWAT $^{90}$ and thus it affects the AT structure. Moreover, it was shown that a midfacial STF injection demonstrates some beneficial effects also on the neighboring aesthetic units connected with another facial AT compartment. $^{91}$

Recently it was proposed to shift the main paradigm of STFs from the neocollagenesis in the dermis to the spatial modification of the fat tissue and activation of adiposederived stem cells (ADSCs). ${ }^{42}$ There are experimental results demonstrating that at least some STFs (or their components) can stimulate the ADSCs in vivo. ${ }^{92}$ Whereas this item was not investigated with a scanning electron microscopy until now, it can be assumed that the sWAT might demonstrate the properties of the fibrous WAT after injection, thus improving the local mechanical properties in the injected area.

Better knowledge of facial fat compartments permits a more nuanced analysis of the aging face and better informs the next steps in restoring contour and facial balance, namely selecting optimal injection materials and the volumes required thereof. Injection materials have historically included fat as a filler and collagen-based products. Synthetic fillers are more contemporary alternatives, including HA-based fillers. ${ }^{93}$ Each STF is different in viscosity, stiffness, affinity for water, and in type and degree of cross-linking. ${ }^{93}$ Different STFs may be used, depending on their respective biophysical properties, to target specific AT compartments or subcompartments in varying depth and location. For example, STFs with higher stiffness and viscosity can be used to build volume and lift tissue in the midface or some deep locations in the face, where visibility is of less concern. STFs with less stiffness, which tend to diffuse or spread, are naturally more useful in superficial areas where the skin appearance is of main importance and the palpability is unwanted, such as the lower eyelids or lips. ${ }^{93}$ 


\section{Fat Grafts Targeting Facial Adipose Tissue}

Injection of an autologous fat graft into the subdermal layer in facial regions was shown to provide significant modification of the dWAT layer, which appeared to have a richer microvascular bed after injection ${ }^{94}$ (-Figs. 5-7). On the other hand, the pericellular fibrosis around adipocytes in injected area was significantly reduced, which can theoretically reduce the mechanical stiffness of the AT in this area. This can lead to a paradoxical situation: fat grafts can indeed immediately replace the lost volume in dWAT, but not really improve its mechanical properties.

The use of fat as a filler has been the subject of much study, including the investigation of the precise volume of injected fat required to achieve an optimal face "lift" effect. A study by Rohrich et al $^{95}$ evaluated the "fill and lift" technique in 100 consecutive face lifts and noted that the following subcompartments are most important to fill prior to lifting the facial structures: the medial and middle superficial fat compartment, the deep medial and lateral cheek fat, and the deep nasolabial fat compartment. ${ }^{95}$ That study analyzed the synergistic effects of fat-grafting individual facial fat subcompartments in conjunction with either a "SMAS ectomy" or "SMAS stacking" technique, using the malar contour and nasolabial fold depth as key outcome measures. The average amount injected was $2 \mathrm{cc}$ per fat compartment, with an average total of $12 \mathrm{cc}$ per face (range: 8-14 cc), and an average of $2 \mathrm{cc}$ in the nasolabial folds bilaterally. The study reported a
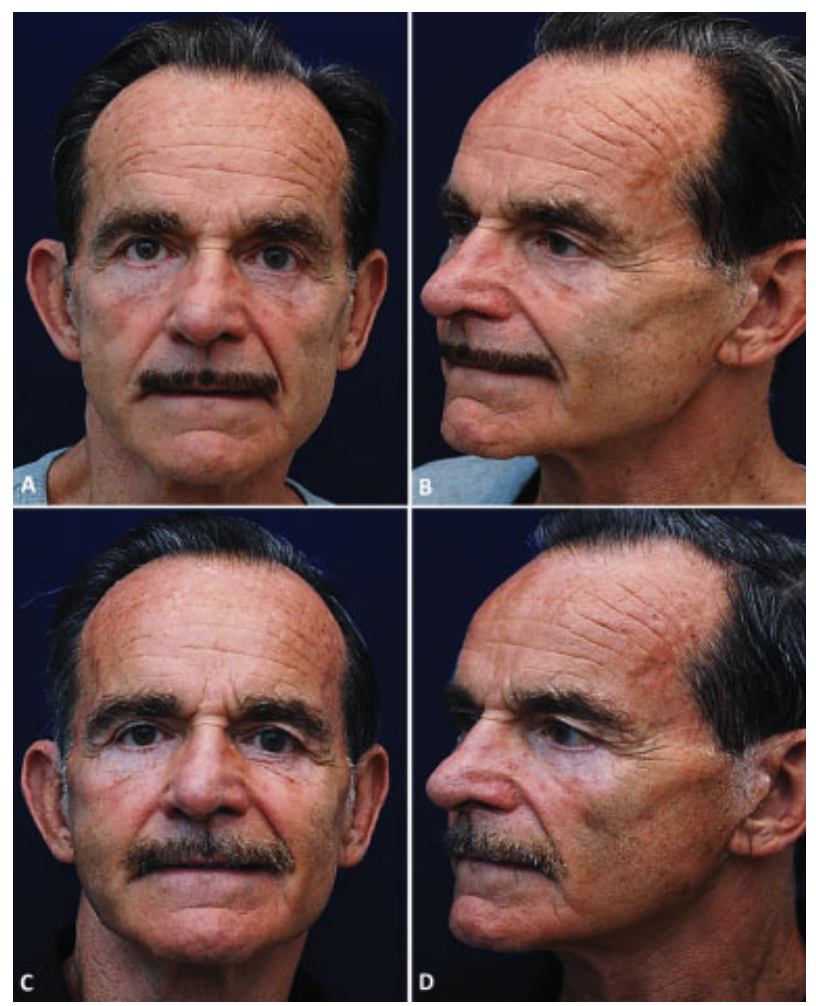

Fig. 5 Before (A, B) and 3 months after (C, D), treatment targeting the suborbicularis oculi fat pad and the deep medial and lateral cheek fat compartments with a 2:1 mixture of autologous fat (centrifuged at 1500RPM for 3 minutes) and platelet-rich fibrin matrix.

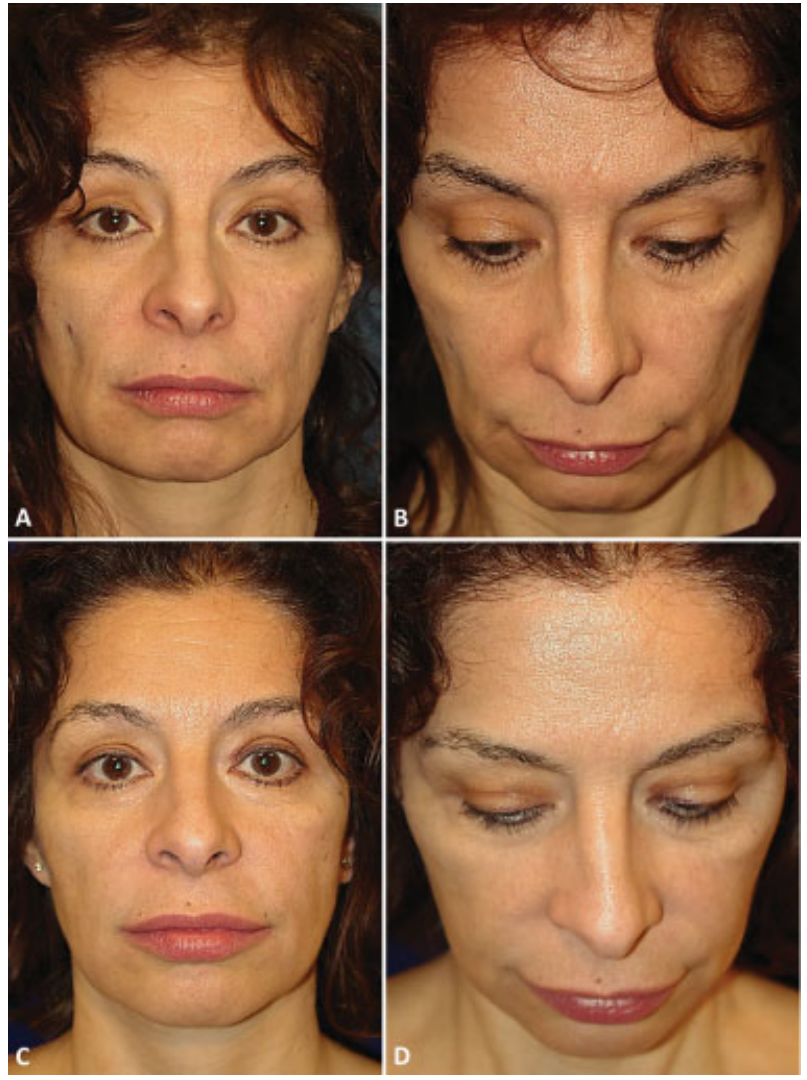

Fig. 6 Before (A, B) and 6 months after (C, D), treatment targeting the nasolabial fold and the deep medial and lateral cheek fat compartments as well as intramuscular injections into orbicularis oris muscle and into the chin fat pad with autologous fat (centrifuged at 1,500 rpm for 3 minutes). Fat was injected inferior to the orbicularis retaining ligament at the infraorbital rim, which helps localize and enhance the projection over the malar eminence.

malar projection increase of approximately $13.5 \%$ and malar tissue lift of approximately $12.2 \%$. The study concluded that the "lift-and-fill" technique effectively addressed the core issue of facial aging, namely volume deflation, and that this technique required less tension to lift, resulting in a more natural appearance. ${ }^{95}$

The perioral region having another WAT structure requires, however, a different approach during augmentation. This area is not typically undermined during a face lift, has superficial fat compartments, is the farthest point from the stretch of the lift, and is surrounded by adherent structures. During the aging process, there is a gradual loss of lip volume and support. Over time, lips become thinner, subcutaneous fat atrophies, nasolabial sulcus deepens, and the labiomandibular sulcus becomes more prominent. A study performed by Pezeshk et al ${ }^{96}$ evaluated and compared 65 patients who underwent rhytidectomy without perioral rejuvenation to 65 patients who underwent rhytidectomy with perioral rejuvenation using fat grafting. The pre- and postoperative photos were evaluated by three independent observers using a modified Fitzpatrick wrinkle scale (0: none, 1: mild, 2: moderate, 3: deep). Fat was harvested, centrifuged, and injected (1-2 cc into each perioral superficial compartment using a 22-gauge needle). Perioral injections 


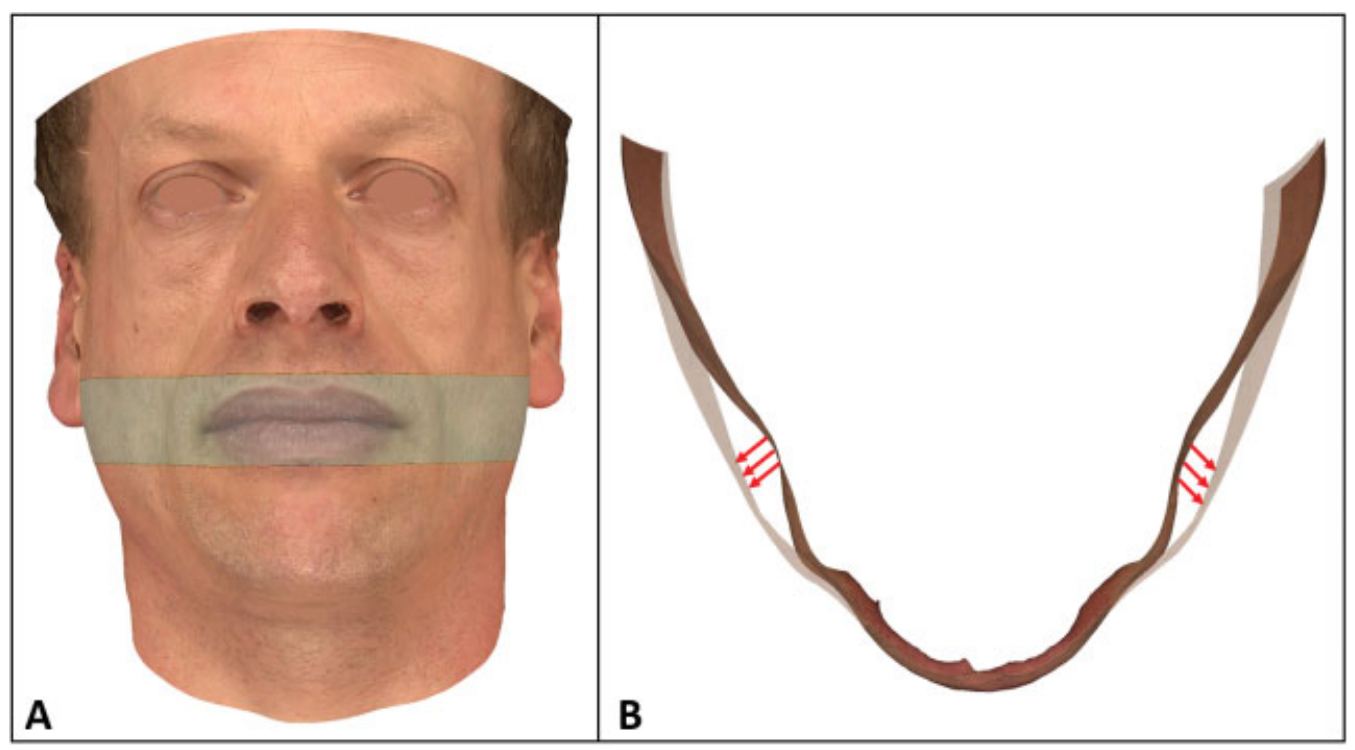

Fig. 7 (A) Preoperative 3D photograph of a 58-year-old man presenting with buccal lipoatrophy. Highlighted in grey is the corresponding area for the later superimposition of pre- and postoperative scans with 15.0-cc autologous lipotransfer. (B) Top viewed pre- and postoperative 3D scans: the translucent scan shows the postoperative result after 4 weeks with 8.4-cc retention. Volume expansion is limited to the injection site (red arrows).

were made in a fan-like manner to create a smooth lateral transition, with central expansion of the lips. Wrinkle scores decreased modestly (1.13-0.74 postoperatively) in the group with no perioral rejuvenation during rhytidectomy, while those patients with perioral rejuvenation improved more significantly (1.61-0.76 postoperatively). The authors emphasized that the face should be considered in three dimensions, taking into consideration the parameters of depth and width, and recommended that this area should be lifted and filled to address the volume depletion that occurs with aging. ${ }^{96}$

Fat has unquestionable value as a filler in restoring volume, contour, and harmony to the face during rejuvenation surgery. Once selected as the appropriate filler to the task at hand, the focus shifts to proper harvesting and integration of the fat during autologous transfer. Multiple harvesting sites in the body have been described, such as the abdomen, flank, thigh, and knee. Recent studies evaluating adipocyte viability show that no harvest site is statistically better than another and that resorption rates after grafting are highly variable, between 20 and $90 \%{ }^{97}$

Subsequent studies have investigated inflammatory changes, as well as the changes that occur in the adipocytes at a cellular level that might influence viability. Suga et $\mathrm{al}^{98}$ investigated changes in mouse AT after ischemia/hypoxia caused by variably restricting blood supply to the AT. This model operated on the fact that the partial pressure in mice is similar to that of humans, measuring approximately 50 to $60 \mathrm{~mm} \mathrm{Hg}$. The study described a series of events in AT, beginning with induction of ischemia, followed by apoptosis of adipocytes, blood-derived cells, and endothelial cells on day 1 after harvesting. By days 3 and 7, the study described necrosis of the ATs, followed by infiltration of macrophages and phagocytosis. These ischemic changes were followed by regenerative actions, including angiogenesis, adipogenesis, and proliferation of cells. Accordingly, the authors suggest that supplementing a fat graft with ADSCs may reduce longterm graft atrophy and scar formation after severe ischemia occurring at the graft site as they were able to survive in the severe ischemia/hypoxia model. ${ }^{98}$

The use of ADSCs in conjunction with AT in fat grafting is a promising technique to provide volume and regenerate overlying, aged, and thinned skin. Further research on the role ADSCs in fat grafting may yield innovative and unprecedented application in treatment of the aging face.

\section{Conclusion}

The facial fat is a heterogeneous entity that can be subdivided into different anatomical compartments with each of them having different adipocyte morphology and different extracellular collagenous matrix. These different compositions provide unique and specific mechanical and histochemical properties to each of the respective compartments. Minimally invasive and surgical interventions aiming to restore the youthful face have to account for the different fat properties during therapeutic applications of STFs or fat grafting. However, still little is known about the macro- and microscopic characteristics of the facial fat tissue and future studies will be needed to reveal new insights to better understand the process of facial aging and how to fight its signs best.

\section{Funding}

No funding was obtained for this work.

\section{Author Disclosures}

IK is the managing partner of Wellcomet GmbH. No methods or devices of Wellcomet $\mathrm{GmbH}$ were used or 
mentioned in this publication. None of the other authors listed have any commercial associations or financial disclosures that might pose or create a conflict of interest with the results presented or methods applied in this study.

\section{Statement}

The facts and citations mentioned in the manuscript are reported to the best knowledge of the authors and potential deviations from the current understanding of the concepts presented should be considered in this narrative review.

\section{References}

1 Jones FW. Man's Place among the Mammals. 1929London, UK: Arnold; 1929

2 Stecco C, Macchi V, Porzionato A, Duparc F, De Caro R. The fascia: the forgotten structure. Ital J Anat Embryol 2011;116(3):127-138

3 Lancerotto L, Stecco C, Macchi V, Porzionato A, Stecco A, De Caro R. Layers of the abdominal wall: anatomical investigation of subcutaneous tissue and superficial fascia. Surg Radiol Anat 2011; 33(10):835-842

4 Wan D, Amirlak B, Giessler P, et al. The differing adipocyte morphologies of deep versus superficial midfacial fat compartments: a cadaveric study. Plast Reconstr Surg 2014;133(5): $615 e-622 e$

5 Cotofana S, Schenck TL, Trevidic P, et al. Midface: clinical anatomy and regional approaches with injectable fillers. Plast Reconstr Surg 2015;136(5, Suppl):219S-234S

6 Pilsl U, Anderhuber F. The chin and adjacent fat compartments. Dermatol Surg 2010;36(2):214-218

7 Pilsl U, Anderhuber F. The septum subcutaneum parotideomassetericum. Dermatol Surg 2010;36(12):2005-2008

8 Rohrich RJ, Pessa JE. The fat compartments of the face: anatomy and clinical implications for cosmetic surgery. Plast Reconstr Surg 2007;119(7):2219-2227, discussion 2228-2231

9 Gierloff M, Stöhring C, Buder T, Wiltfang J. The subcutaneous fat compartments in relation to aesthetically important facial folds and rhytides. J Plast Reconstr Aesthet Surg 2012;65(10):1292-1297

10 Stuzin JM, Baker TJ, Gordon HL. The relationship of the superficial and deep facial fascias: relevance to rhytidectomy and aging. Plast Reconstr Surg 1992;89(3):441-449, discussion 450-451

11 Ghassemi A, Prescher A, Riediger D, Axer H. Anatomy of the SMAS revisited. Aesthetic Plast Surg 2003;27(4):258-264

12 Bertossi D, Bernardi P, Benati D, Ruffoli M, Sbarbati A, Nocini P. Classification of fat pad of the third medium of the face. Aesth Med 2015;1(3):103-109

13 Cotofana S, Fratila AA, Schenck TL, Redka-Swoboda W, Zilinsky I, Pavicic T. The anatomy of the aging face: a review. Facial Plast Surg 2016;32(3):253-260

14 Mendelson BC, Freeman ME, Wu W, Huggins RJ. Surgical anatomy of the lower face: the premasseter space, the jowl, and the labiomandibular fold. Aesthetic Plast Surg 2008;32(2):185-195

15 Mendelson $\mathrm{BC}$, Wong $\mathrm{CH}$. Surgical anatomy of the middle premasseter space and its application in sub-SMAS face lift surgery. Plast Reconstr Surg 2013;132(1):57-64

16 Wong $\mathrm{CH}$, Mendelson B. Facial soft-tissue spaces and retaining ligaments of the midcheek: defining the premaxillary space. Plast Reconstr Surg 2013;132(1):49-56

17 Rohrich RJ, Arbique GM, Wong C, Brown S, Pessa JE. The anatomy of suborbicularis fat: implications for periorbital rejuvenation. Plast Reconstr Surg 2009;124(3):946-951
18 Rohrich RJ, Pessa JE, Ristow B. The youthful cheek and the deep medial fat compartment. Plast Reconstr Surg 2008;121(6): 2107-2112

19 Aghai F, Caix P. [The retro-orbicularis oculus fat (ROOF) or Charpy's fat pad. Descriptive and functional anatomy. Surgical concepts applied to the design of a frontotemporal lift procedure] [in French]. Ann Chir Plast Esthet 2004;49(4):355-359

20 Aiache $\mathrm{AE}$, Ramirez $\mathrm{OH}$. The suborbicularis oculi fat pads: an anatomic and clinical study. Plast Reconstr Surg 1995;95(1):37-42

21 Gierloff M, Stöhring C, Buder T, Gassling V, Açil Y, Wiltfang J. Aging changes of the midfacial fat compartments: a computed tomographic study. Plast Reconstr Surg 2012;129(1):263-273

22 Surek CK, Vargo J, Lamb J. Deep pyriform space: anatomical clarifications and clinical implications. Plast Reconstr Surg 2016; 138(1):59-64

23 Mendelson BC, Muzaffar AR, Adams WP Jr. Surgical anatomy of the midcheek and malar mounds. Plast Reconstr Surg 2002;110(3): 885-896, discussion 897-911

24 Bichat MFX. Anatomie Generale appliquèe a la Physiologie at a la Medecine. Anatomie Gen 1801;1:202

25 Zenker W. [New findings in temporal muscle in man] [in German, Russian]. Z Anat Entwicklungsgesch 1955;118(4):355-368

26 O'Brien JX, Ashton MW, Rozen WM, Ross R, Mendelson BC. New perspectives on the surgical anatomy and nomenclature of the temporal region: literature review and dissection study. Plast Reconstr Surg 2013;131(3):510-522

27 Andretto Amodeo C, Casasco A, Icaro C A, Kang R, Keller GS. The suborbicularis oculi fat (SOOF) and the fascial planes: has everything already been explained? JAMA Facial Plast Surg 2014;16(1): $36-41$

28 Spalding KL, Arner E, Westermark PO, et al. Dynamics of fat cell turnover in humans. Nature 2008;453(7196):783-787

29 Arner E, Westermark PO, Spalding KL, et al. Adipocyte turnover: relevance to human adipose tissue morphology. Diabetes 2010; 59(1):105-109

30 Festa E, Fretz J, Berry R, et al. Adipocyte lineage cells contribute to the skin stem cell niche to drive hair cycling. Cell 2011;146(5):761-771

31 Schmidt BA, Horsley V. Intradermal adipocytes mediate fibroblast recruitment during skin wound healing. Development 2013; 140(7):1517-1527

32 Kasza I, Suh Y, Wollny D, et al. Syndecan-1 is required to maintain intradermal fat and prevent cold stress. PLoS Genet 2014;10(8): e1004514

33 Alexander CM, Kasza I, Yen CL, et al. Dermal white adipose tissue: a new component of the thermogenic response. J Lipid Res 2015; 56(11):2061-2069

34 Zhang LJ, Guerrero-Juarez CF, Hata T, et al. Innate immunity. Dermal adipocytes protect against invasive Staphylococcus aureus skin infection. Science 2015;347(6217):67-71

35 Kruglikov IL, Scherer PE. Dermal adipocytes: from irrelevance to metabolic targets? Trends Endocrinol Metab 2016;27(1):1-10

36 Kruglikov IL, Scherer PE, Wollina U. Are dermal adipocytes involved in psoriasis? Exp Dermatol 2016;25(10):812-813

37 Brembilla NC, Boehncke WH. Dermal adipocytes' claim for fame in psoriasis. Exp Dermatol 2016

38 Kruglikov IL, Scherer PE. Skin aging: are adipocytes the next target? Aging (Albany, NY) 2016;8(7):1457-1469

39 Marangoni RG, Korman BD, Wei J, et al. Myofibroblasts in murine cutaneous fibrosis originate from adiponectin-positive intradermal progenitors. Arthritis Rheumatol 2015;67(4):1062-1073

40 Driskell RR, Jahoda CA, Chuong CM, Watt FM, Horsley V. Defining dermal adipose tissue. Exp Dermatol 2014;23(9):629-631

41 Schneider MR. Coming home at last: dermal white adipose tissue. Exp Dermatol 2014;23(9):634-635

42 Kruglikov IL, Wollina U. Soft tissue fillers as non-specific modulators of adipogenesis: change of the paradigm? Exp Dermatol 2015;24(12):912-915 
43 Kruglikov IL, Scherer PE. Dermal adipocytes and hair cycling: is spatial heterogeneity a characteristic feature of the dermal adipose tissue depot? Exp Dermatol 2016;25(4):258-262

44 Engrav LH, Tuggle CK, Kerr KF, et al. Functional genomics unique to week 20 post wounding in the deep cone/fat dome of the Duroc/ Yorkshire porcine model of fibroproliferative scarring. PLoS One 2011;6(4):e19024

45 Eto H, Suga H, Matsumoto D, et al. Characterization of structure and cellular components of aspirated and excised adipose tissue. Plast Reconstr Surg 2009;124(4):1087-1097

46 Tchoukalova YD, Koutsari C, Karpyak MV, Votruba SB, Wendland E, Jensen MD. Subcutaneous adipocyte size and body fat distribution. Am J Clin Nutr 2008;87(1):56-63

47 Tchoukalova YD, Koutsari C, Votruba SB, et al. Sex- and depotdependent differences in adipogenesis in normal-weight humans. Obesity (Silver Spring) 2010;18(10):1875-1880

48 White UA, Tchoukalova YD. Sex dimorphism and depot differences in adipose tissue function. Biochim Biophys Acta 2014;1842(3): 377-392

49 McLaughlin T, Lamendola C, Coghlan N, et al. Subcutaneous adipose cell size and distribution: relationship to insulin resistance and body fat. Obesity (Silver Spring) 2014;22(3):673-680

50 Pellegrinelli V, Carobbio S, Vidal-Puig A. Adipose tissue plasticity: how fat depots respond differently to pathophysiological cues. Diabetologia 2016;59(6):1075-1088

51 Wang QA, Tao C, Gupta RK, Scherer PE. Tracking adipogenesis during white adipose tissue development, expansion and regeneration. Nat Med 2013;19(10):1338-1344

52 Hanamoto T, Kajita K, Mori I, et al. The role of small proliferative adipocytes in the development of obesity: comparison between Otsuka Long-Evans Tokushima Fatty (OLETF) rats and non-obese Long-Evans Tokushima Otsuka (LETO) rats. Endocr J 2013;60(8): 1001-1011

53 Sun K, Kusminski CM, Scherer PE. Adipose tissue remodeling and obesity. J Clin Invest 2011;121(6):2094-2101

54 Sun K, Tordjman J, Clément K, Scherer PE. Fibrosis and adipose tissue dysfunction. Cell Metab 2013;18(4):470-477

55 Sun K, Park J, Gupta OT, et al. Endotrophin triggers adipose tissue fibrosis and metabolic dysfunction. Nat Commun 2014;5:3485

56 Buechler C, Krautbauer S, Eisinger K. Adipose tissue fibrosis. World J Diabetes 2015;6(4):548-553

57 Khan T, Muise ES, Iyengar P, et al. Metabolic dysregulation and adipose tissue fibrosis: role of collagen VI. Mol Cell Biol 2009; 29(6):1575-1591

58 Divoux A, Tordjman J, Lacasa D, et al. Fibrosis in human adipose tissue: composition, distribution, and link with lipid metabolism and fat mass loss. Diabetes 2010;59(11):2817-2825

59 Nakajima I, Muroya S, Tanabe R, Chikuni K. Extracellular matrix development during differentiation into adipocytes with a unique increase in type V and VI collagen. Biol Cell 2002;94(3):197-203

60 Pasarica M, Gowronska-Kozak B, Burk D, et al. Adipose tissue collagen VI in obesity. J Clin Endocrinol Metab 2009;94(12): 5155-5162

61 Han CY, Subramanian S, Chan CK, et al. Adipocyte-derived serum amyloid A3 and hyaluronan play a role in monocyte recruitment and adhesion. Diabetes 2007;56(9):2260-2273

62 Kruglikov I. Biophysical basics of body treatments. Is hyaluronan a link that has gone unnoticed? Am J Cosmet Surg 2012;29(2): 121-127

63 Ceperuelo-Mallafré V, Miranda M, Chacón MR, et al. Adipose tissue expression of the glycerol channel aquaporin-7 gene is altered in severe obesity but not in type 2 diabetes. J Clin Endocrinol Metab 2007;92(9):3640-3645

64 Kang L, Lantier L, Kennedy A, et al. Hyaluronan accumulates with high-fat feeding and contributes to insulin resistance. Diabetes 2013;62(6):1888-1896
65 Zhu Y, Crewe C, Scherer PE. Hyaluronan in adipose tissue: beyond dermal filler and therapeutic carrier. Sci Transl Med 2016;8(323): $323 \mathrm{ps} 4$

66 Kruglikov IL. Is the depletion of hyaluronan in hypertrophic fat tissue a key event in body-contouring procedures? Am J Cosmet Surg 2013;30(4):244-245

67 Li Y, Rahmanian M, Widström C, Lepperdinger G, Frost GI, Heldin P. Irradiation-induced expression of hyaluronan (HA) synthase 2 and hyaluronidase 2 genes in rat lung tissue accompanies active turnover of HA and induction of types I and III collagen gene expression. Am J Respir Cell Mol Biol 2000;23(3):411-418

68 Kruglikov I. The pathophysiology of cellulite: can the puzzle eventually be solved? JCDSA 2012;2(1):1-7

69 Kielty CM, Whittaker SP, Grant ME, Shuttleworth CA. Type VI collagen microfibrils: evidence for a structural association with hyaluronan. J Cell Biol 1992;118(4):979-990

70 Wan D, Amirlak B, Rohrich R, Davis K. The clinical importance of the fat compartments in midfacial aging. Plast Reconstr Surg Glob Open 2014;1(9):e92

71 Sbarbati A, Accorsi D, Benati D, et al. Subcutaneous adipose tissue classification. Eur J Histochem 2010;54(4):e48

72 Comley K, Fleck NA. The toughness of adipose tissue: measurements and physical basis. J Biomech 2010;43(9):1823-1826

73 Kruglikov IL. General theory of body contouring: 2. Modulation of mechanical properties of subcutaneous fat tissue. JCDSA 2014; 4(1):117-127

74 Alkhouli N, Mansfield J, Green E, et al. The mechanical properties of human adipose tissues and their relationships to the structure and composition of the extracellular matrix. Am J Physiol Endocrinol Metab 2013;305(12):E1427-E1435

75 Lackey DE, Burk DH, Ali MR, et al. Contributions of adipose tissue architectural and tensile properties toward defining healthy and unhealthy obesity. Am J Physiol Endocrinol Metab 2014;306(3): E233-E246

76 Bilkei-Gorzo A, Drews E, Albayram Ö, et al. Early onset of aging-like changes is restricted to cognitive abilities and skin structure in Cnr1 ${ }^{-} /^{-}$mice. Neurobiol Aging 2012;33(1):200.e11-200.e22

77 Mitani H, Koshiishi I, Toyoda H, Toida T, Imanari T. Alterations of hairless mouse skin exposed to chronic UV irradiation and its prevention by hydrocortisone. Photochem Photobiol 1999;69(1): 41-46

78 Sayama A, Soushin T, Okada T, Doi K, Nakayama H. Morphological and biochemical changes during aging and photoaging of the skin of C57BL/6J mice. J Toxicol Pathol 2010;23(3):133-139

79 Ezure T, Amano S. Negative regulation of dermal fibroblasts by enlarged adipocytes through release of free fatty acids. J Invest Dermatol 2011;131(10):2004-2009

80 Ezure T, Amano S. Increment of subcutaneous adipose tissue is associated with decrease of elastic fibres in the dermal layer. Exp Dermatol 2015;24(12):924-929

81 Sherratt MJ. Tissue elasticity and the ageing elastic fibre. Age (Dordr) 2009;31(4):305-325

82 Sherratt MJ. Body mass index and dermal remodelling. Exp Dermatol 2015;24(12):922-923

83 Kruglikov IL. Neocollagenesis in non-invasive aesthetic treatments. JCDSA 2013;3(1A):1-5

84 Ezure T, Hosoi J, Amano S, Tsuchiya T. Sagging of the cheek is related to skin elasticity, fat mass and mimetic muscle function. Skin Res Technol 2009;15(3):299-305

85 Ezure T, Amano S. Influence of subcutaneous adipose tissue mass on dermal elasticity and sagging severity in lower cheek. Skin Res Technol 2010;16(3):332-338

86 Ezure T, Amano S. Involvement of upper cheek sagging in nasolabial fold formation. Skin Res Technol 2012;18(3):259-264

87 Pessa JE, Nguyen H, John GB, Scherer PE. The anatomical basis for wrinkles. Aesthet Surg J 2014;34(2):227-234 
88 Rutkowski JM, Davis KE, Scherer PE. Mechanisms of obesity and related pathologies: the macro- and microcirculation of adipose tissue. FEBS J 2009;276(20):5738-5746

89 Wollina U. Midfacial rejuvenation by hyaluronic acid fillers and subcutaneous adipose tissue-a new concept. Med Hypotheses 2015;84(4):327-330

90 Arlette JP, Trotter MJ. Anatomic location of hyaluronic acid filler material injected into nasolabial fold: a histologic study. Dermatol Surg 2008;34(1, Suppl 1):S56-S62, discussion S62-S63

91 Wollina U. Facial rejuvenation starts in the midface: three-dimensional volumetric facial rejuvenation has beneficial effects on nontreated neighboring esthetic units. J Cosmet Dermatol 2016; 15(1):82-88

92 Huang SH, Lin YN, Lee SS, et al. New adipose tissue formation by human adipose-derived stem cells with hyaluronic acid gel in immunodeficient mice. Int J Med Sci 2015;12(2):154-162

93 Ramanadham SR, Rohrich RJ. Newer understanding of specific anatomic targets in the aging face as applied to injectables: superficial and deep facial fat compartments-an evolving target for site-specific facial augmentation. Plast Reconstr Surg 2015;136 (5, Suppl):49S-55S

94 Charles-de-Sá L, Gontijo-de-Amorim NF, Maeda T C, et al. Antiaging treatment of the facial skin by fat graft and adipose-derived stem cells. Plast Reconstr Surg 2015;135(4):999-1009

95 Rohrich RJ, Ghavami A, Constantine FC, Unger J, Mojallal A. Liftand-fill face lift: integrating the fat compartments. Plast Reconstr Surg 2014;133(6):756e-767e

96 Pezeshk RA, Stark RY, Small KH, Unger JG, Rohrich RJ. Role of autologous fat transfer to the superficial fat compartments for perioral rejuvenation. Plast Reconstr Surg 2015;136(3): 301e-309e

97 Rohrich RJ, Sorokin ES, Brown SA. In search of improved fat transfer viability: a quantitative analysis of the role of centrifugation and harvest site. Plast Reconstr Surg 2004;113(1):391-395, discussion 396-397

98 Suga H, Eto $\mathrm{H}$, Aoi N, et al. Adipose tissue remodeling under ischemia: death of adipocytes and activation of stem/progenitor cells. Plast Reconstr Surg 2010;126(6):1911-1923 